\title{
A Novel Surgical Technique: Single-Incision Transumbilical Laparoscopic Roux-en-Y Gastric Bypass
}

\author{
Chih-Kun Huang • Sheng-Fa Yao • Chi-Hsien Lo • \\ Jer-Yiing Houng $\cdot$ Yaw-Sen Chen $\cdot$ Po-Huang Lee
}

Published online: 1 July 2010

(C) The Author(s) 2010. This article is published with open access at Springerlink.com

\begin{abstract}
Conventional laparoscopic Roux-en-Y gastric bypass (LRYGB) is a gold standard for bariatric surgery, but the procedure requires five to seven incisions for placement of multiple trocars and thus may produce lessthan-ideal cosmetic results. We have developed a new approach, single-incision transumbilical LRYGB (SITULRYGB) to treat morbid obesity. We compared the surgical results and patient satisfaction in a study of five-port LRYGB and SITU-LRYGB. Fifty morbidly obese patients (14 males, 36 females) underwent either Roux-en-Y gastric bypass with five-port LRYGB or the SITU-LRYGB approach. During the operation, we used a novel intraoperative liver traction method with a "liver suspension tape" that we specifically designed for SITU-LRYGB. Compared to five-port surgery with SITU-LRYGB, there were no intraoperative complications, wound healing was
\end{abstract}

C.-K. Huang • C.-H. Lo

Bariatric \& Metabolic International Surgery Center,

E-Da Hospital,

Kaohsiung, Taiwan

C.-K. Huang

e-mail: dr.ckhuang@hotmail.com

C.-K. Huang • Y.-S. Chen • P.-H. Lee

Department of General Surgery, E-Da Hospital,

Kaohsiung, Taiwan

C.-K. Huang $•$ J.-Y. Houng $(\bowtie)$

Department of Chemical Engineering,

Institute of Biotechnology and Chemical Engineering,

I-Shou University,

E-Da Rd, Jian-Shu Tsuen, Yan-Chau Shiang,

Kaohsiung 824, Taiwan

e-mail: jyhoung@isu.edu.tw

S.-F. Yao

Department of Plastic Surgery, E-Da Hospital,

Kaohsiung, Taiwan excellent, and there was no abdominal scarring. SITU surgical time was longer than that with five-port LRYGB ( 99.8 vs. $67.6 \mathrm{~min}, P<0.001$ ). Patients treated with the fiveport method were more obese than those in the SITU group (127.9 vs. $112.4 \mathrm{~kg}, P=0.016$ ). After the bariatric surgery, no difference in comorbidity was found in both groups. Patient satisfaction was greater with SITU than with the five-port method (4.48 vs. 3.96, $P=0.006$ ). Roux-en-Y gastric bypass can be successfully achieved via a single umbilical incision, a method that provides a short operative time and good recovery and eliminates abdominal scarring.

Keywords Laparoscopic Roux-en-Y gastric bypass .

Single-incision transumbilical laparoscopic surgery - SILS . Gastric bypass · Laparoscopy $\cdot$ Bariatric surgery

\section{Introduction}

Laparoscopic Roux-en-Y gastric bypass (LRYGB) has become one of the most popular approaches for bariatric surgery throughout Asia. Most investigators have reported that patients lose $60 \%$ to $70 \%$ of excess body weight after undergoing this procedure and that these patients maintain their weight loss for more than 10 years [1-4]. However, LRYGB is a complex type of bariatric surgery and has a steep learning curve, perhaps more than many other advanced laparoscopic procedures [5].

One drawback of the laparoscopic technique is that it requires five to seven abdominal incisions to facilitate placement of the multiple trocars used during the procedure. Because of the need for the numerous ports, there is often a poor cosmetic result. These port site wounds have visible scars that may fade over time; however, the healing process is highly individualistic, and the cosmetic outcome may not be appealing to all patients, at least during the 
early postoperative period. And most Asian patients have pigmentation of the scar after healing. Nevertheless, since Wittgrove et al. introduced the laparoscopic technique in 1994, the number of operations performed has grown rapidly, and there has been a gradual reduction in the number of complications and better cosmetic results $[6,7]$.

Newer laparoscopic approaches have eliminated the need for multiple ports and the inevitable scarring that follows. Natural orifice transluminal endoscopic surgery (NOTES), which produces no scarring, has been considered to be a landmark in the advancement of laparoscopy. Since the introduction of NOTES in 2004, numerous reports have described its use for various surgical interventions [8-10]. Although it has attracted the attention of surgeons worldwide, the techniques and instruments used for this new and minimally invasive procedure are still very much in the developmental stage. Recently, an emerging procedure, single-incision laparoscopic surgery (SILS), has been used for cholecystectomy, appendectomy, adjustable gastric banding, and sleeve gastrectomy [11-16]. In bariatric surgeries, the SILS technique has been employed to perform adjustable banding and laparoscopic sleeve gastrectomy because both procedures require extension of oneport wound for placement of the band or extraction of the specimen. The single incision in the SILS technique easily allows for these without producing an additional scar [1719]. And although new ports and instruments are becoming more readily available for single-incision surgery, they are not yet licensed for use in Taiwan.

SILS performed via an umbilical incision produces a better cosmetic outcome than does five- to seven-port laparoscopic bariatric surgery because the surgical wound can be hidden in the umbilicus, leaving no visible abdominal scars [18]. Despite these advantages over the more traditional procedure, the small umbilical incision "crowds" the trocars, and the surgeon has only a small angle in which to work. Furthermore, in morbidly obese patients, the hypertrophic left liver lobe invariably hinders the surgeon's view of the entire stomach. Consequently, traction of the liver while instruments are manipulated during this surgery is a major concern.

We have developed an approach to bariatric surgery that uses a modified form of LRYGB. In our single-incision transumbilical laparoscopic Roux-en-Y gastric bypass (SITU-LRYGB) technique, we have refined conventional LRYGB to include the benefits of SILS with improved visualization and working space during surgery. This new technique has several advantages over previous approaches for treating patients with morbid obesity. Our group reported the first case using this method, which showed an acceptable operation time and much better cosmetic results [19]. The operation requires a special approach for counter-traction that includes special modifications, includ- ing the use of a tape to suspend the liver and stay traction for repair of the jejunojejunostoma. However, we found that overcoming the problem of crowded trocars in a $4-\mathrm{cm}$ umbilical wound is one of the greatest challenges for bariatric surgeons performing this complex surgery. Thus, we developed a new method with extension of the omega wound to $6 \mathrm{~cm}$ to facilitate the surgical process, and we perform subsequent umbilicoplasty to hide the umbilical scar. Patient selection is also important, and the procedure is not recommended for extremely obese or extremely tall patients.

In this article, we compare the results of SITU-LRYGB to five-port LRYGB in two groups of morbidly obese patients. In addition, we outline novel intraoperative liver traction and omega-umbilicoplasty methods.

\section{Methods}

Patients

From November 2008 to May 2009, 50 morbidly obese patients (14 males, 36 females) underwent either Roux-en$\mathrm{Y}$ gastric bypass with five-port or the SITU approach. Surgical criteria for morbid obesity were based on the National Institutes of Health Consensus Development Panel report of 1991, including patients with a body mass index (BMI) of $35 \mathrm{~kg} / \mathrm{m}^{2}$ with comorbidity or a BMI of $40 \mathrm{~kg} / \mathrm{m}^{2}$ with or without comorbidities. The patients ranged in age from 19 to 46 years (mean age 29.8 years) and had BMIs ranging from 35.3 to $55.3 \mathrm{~kg} / \mathrm{m}^{2}$ (mean $43.6 \mathrm{~kg} / \mathrm{m}^{2}$ ). Patients were asked to select the type of surgery they wanted, but super-obese patients (BMIs greater than $60 \mathrm{~kg} / \mathrm{m}^{2}$ ) were excluded from receiving SITU-LRYGB. To measure patients' satisfaction with their operation, a wound satisfaction questionnaire was given to patients 3 months after their operation (Table 1).

\section{Surgical Procedures}

\section{SITU-LRYGB Technique}

All 25 patients were placed in the supine position with their arms extended laterally. The surgeon stood on the right side of the patient and the assistant on the left side. Then, an omega-shaped $6.0-\mathrm{cm}$ incision was made around the upper half of the umbilicus (Fig. 1).

Table 1 Patient satisfaction score

\begin{tabular}{ccccc}
$\begin{array}{l}\text { Very } \\
\text { unsatisfied }\end{array}$ & Unsatisfied & Acceptable & Satisfied & Very satisfied \\
\hline 1 & 2 & 3 & 4 & 5 \\
\hline
\end{tabular}




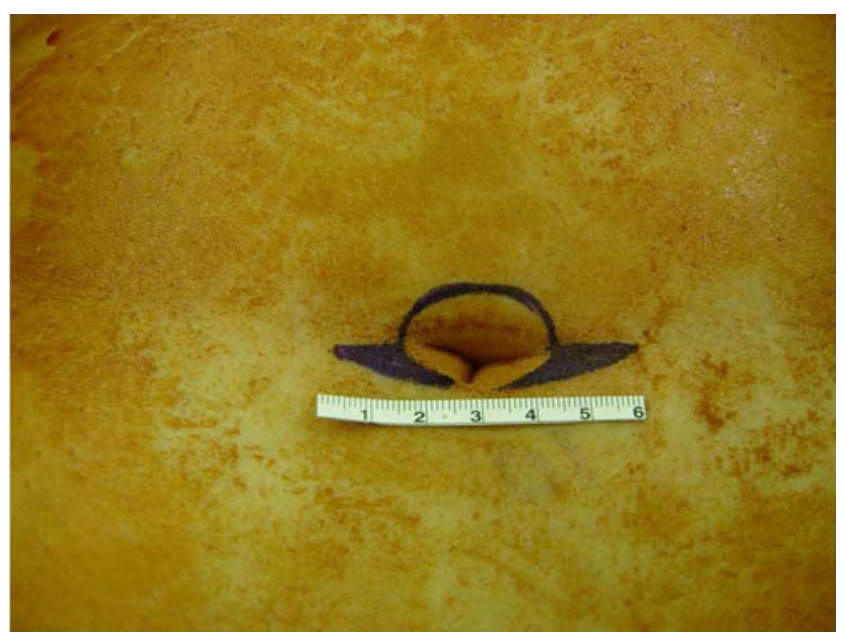

Fig. 1 Omega-shaped 6-cm skin incision in SITU-LRYGB. The blue area will be excised during a plasty process after the initial operation

We then deepened the incision to the linea alba, where an ENDOPATH ${ }^{\circledR} \mathrm{XCEL}^{\mathrm{TM}}$ (Ethicon ${ }^{\circledR}$ Endo-Surgery, Cincinnati, OH, USA) and 12-mm ENDOPATH Bladeless Trocar $^{\mathrm{TM}}$ (Ethicon, Somerville, NJ, USA) were inserted under direct vision with a $5-\mathrm{mm}$, rigid, $30^{\circ}$ video laparoscope. Then, carbon dioxide insufflation was used to create a pneumoperitoneal pressure of $15 \mathrm{mmHg}$. Under direct visualization, the other two 5- and 12-mm ENDOPATH XCEL Bladeless Trocars ${ }^{\mathrm{TM}}$ (Ethicon) were placed through the bilateral "arms" of the incision. The three trocars were arranged in a triangular grouping (Fig. 2a).

\section{Design for the "Liver Suspension Tape"}

We cut the Jackson-Pratt drain tube into a 6-cm length at the drainage hole site. We then pierced the drainage tube with 2-0 Prolene suture (Monofilament Polypropylene
Suture $\mathrm{W} 8400^{\mathrm{TM}}$, Ethicon), according to the diameter of the hole. Needles were left in both sides for further liver puncture. After this, we placed our liver suspension tape into the peritoneal cavity. One needle was held by the needle holder and advanced until it penetrated the left liver near the falciform ligament; the needle was then brought out from the midline abdominal wall. Another needle penetrated the lateral edge of the left liver and was then brought out from the left subcostal abdominal wall. Then the liver was suspended in its proper position and the sutures were fixed with a Kelly clamp.

A harmonic scalpel (Ethicon) was used to dissect the perigastric vessels. Then we created a $25-\mathrm{ml}$ proximal gastric pouch with ENDOPATH ${ }^{\circledR}$ ETS Long45A (Endoscopic Linear Cutters 6R45B ${ }^{\mathrm{TM}}$, Ethicon). Next, the proximal jejunum was traced and measured $100 \mathrm{~cm}$ distal to the ligament of Treitz. It was brought up to create a $2.5-\mathrm{cm}$-long gastrojejunostomy with an endoscopic linear cutter (6R $45 \mathrm{~B}^{\mathrm{TM}}$, Ethicon) at the antecolic position. Then, the proximal jejunum was transected with endoscopic linear cutters (TR45WTM, Ethicon) just near the gastrojejunostomy site and then brought down to perform a side-to-side jejunojejunostomy, with a 100 -cm alimentary limb, by using an endoscopic linear cutter (ENDOPATH ETS ${ }^{\mathrm{TM}}$, Ethicon). Then we placed three stay sutures to retract the jejunojejunostomy, and the stoma was closed with endoscopic linear cutters $\left(6 \mathrm{R} 45 \mathrm{~B}^{\mathrm{TM}}\right.$, Ethicon). In this technique, no articulating instruments were needed. And because we used a harmonic scalpel and Endocutter, we needed no assistants. Use of the liver suspension tape also enabled us to lift the liver without assistance.

The mesenteric defect was closed with 2-0 polybutylatecoated, braided polyester sutures (Ethibond ${ }^{\mathrm{TM}}$ W6977, Ethicon). The gastrojejunostoma was closed with one layer of 2-0 Vicryl PLUS ${ }^{\mathrm{TM}}$ antibacterial sutures (VCP333 ${ }^{\mathrm{TM}}$,
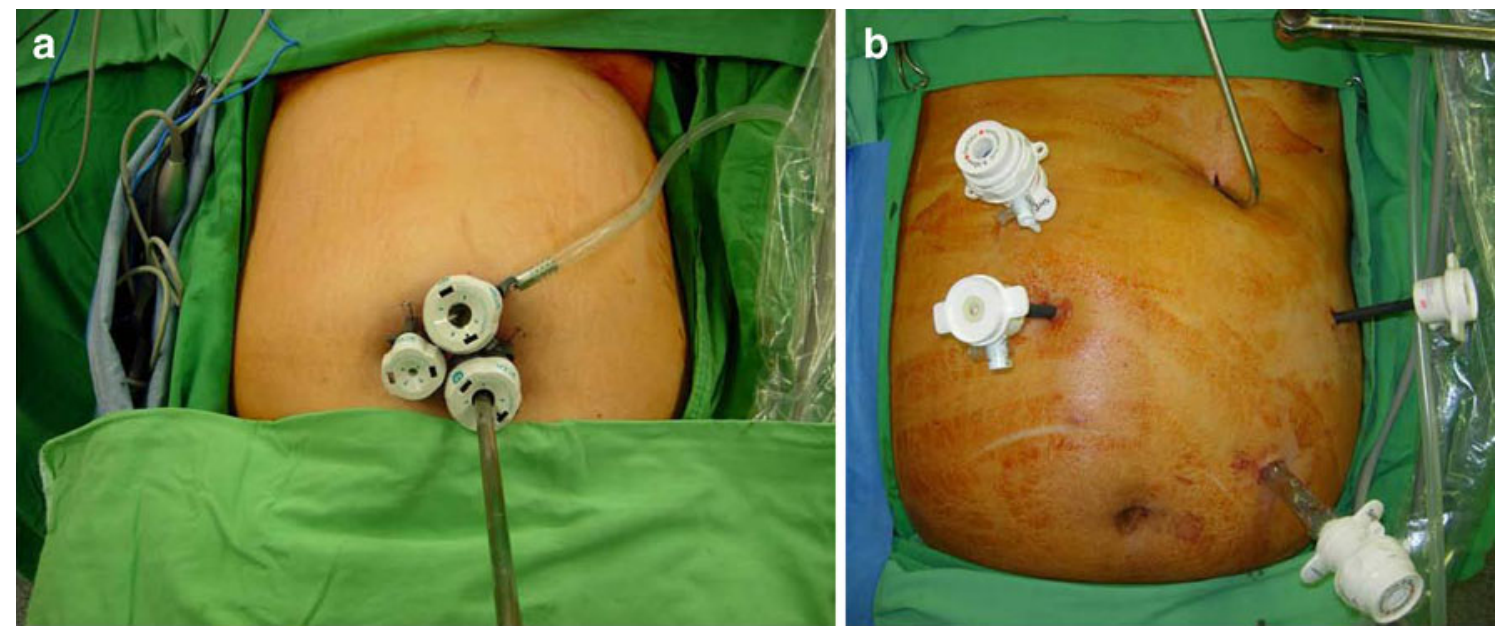

Fig. 2 a Triangular position of trocars in SITU-LRYGB. b The position of trocars and liver retractor in five-port LRYGB 
Ethicon). After this, the liver suspension tape was removed, and homeostasis was achieved by cauterization if hemorrhaging was noted on the liver surface. All trocars were removed and the three fasciae defect was closed with sutures individually.

The original $6.0-\mathrm{cm}$ omega-shaped umbilical wound was revised into a $3.5-\mathrm{cm}$ circular wound around the umbilicus. Finally, a dressing was applied to the skin incision.

\section{Five-Port LRYGB Technique}

The patient was placed in the supine position, and general anesthesia was administered. The surgeon stood on the right side of the patient and the assistant on the left side. A pneumoperitoneum was created by puncturing the peritoneal cavity with a Veress needle in the left periumbilical area. Four ports were created in the usual manner. A liver retractor was introduced at the subxiphoid area (Fig. 2b). A grasper was used to hold the stomach and the Endocutter was used to divide the gastric pouch. We created a $25-\mathrm{ml}$ proximal gastric pouch with the $60-\mathrm{mm}$ linear cutter and the $3.5-\mathrm{mm}$ stapler. We performed gastrojejunostomy with a $30-\mathrm{mm}$ linear cutter and a $2.5-\mathrm{mm}$ stapler (Endo-GIA, Covidien) at the antecolic position. Next, the proximal jejunum was traced and measured $100 \mathrm{~cm}$ distal to the ligament of Treitz. The gastrojejunostomy was $2.5 \mathrm{~cm}$ long. The proximal jejunum was transected just near the gastrojejunostomy site and then brought down to perform a sideto-side jejunojejunostomy using a $60-\mathrm{mm}$ linear cutter and 3.5-mm stapler (Endo-GIA, Covidien). The jejunojejunostoma was closed with the Endo-GIA stapler. Mesenteric defects were closed with braided polyester sutures (2-0
Ethibond, Ethicon, UK). The gastrojejunostoma was closed with sutures (2-0 Vicryl, Ethicon). Then the abdominal wound was repaired primarily after the trocars were removed.

\section{Statistical Analysis}

The mean and SD were computed for continuous variables and evaluated by independent $t$ test. Numbers and percentages were given for categorical variables. The chi-square test was performed to test the association between two categorical variables. When $20 \%$ of cells had expected values $<5$, Fisher's exact test was used instead. All statistics were two-sided and were calculated with SPSS statistical software (version 15.0, SPSS Inc., Chicago, IL, USA). A $P$ value $<0.05$ was considered statistically significant.

\section{Results}

After excluding the first patient [19], who had a 4-cm-long wound and received SITU-LRYGB for $180 \mathrm{~min}$, a total of 50 patients (36 females $(72 \%)$ and 14 males $(28 \%)$ ) were included in the analysis. The average age and BMI were $29.88(\mathrm{SD}=6.36$, range 19 to 46$)$ years and $43.61(\mathrm{SD}=$ 5.21 , range 36.2 to 55.3$) \mathrm{kg} / \mathrm{m}^{2}$.

Table 2 shows the baseline characteristics of the 50 patients. The two groups were similar in age, height, distribution of gender, and prevalence of comorbidities. The main comorbidities in both groups were hyperlipidemia ( $44 \%$ in the SITU group and $32 \%$ in the five-port group), nonalcoholic steatohepatitis $32 \%$ in the SITU group and
Table 2 Characteristics of 50 patients

Continuous variables were presented as mean \pm standard deviation and categorical variables were presented as number (percent)

SITU single-incision transumbilical

${ }^{*} P<0.05$ (statistically significant difference between the two groups)

${ }^{\text {a }} P$ values were calculated by chi-square test

${ }^{\mathrm{b}} P$ values were calculated by independent $t$ test

${ }^{\mathrm{c}} P$ values were calculated by Fisher's exact test

\begin{tabular}{llll}
\hline & \multicolumn{2}{l}{ Surgical method } & $P$ \\
\cline { 2 - 4 } & SITU-LRYGB $(n=25)$ & 5-port LRYGB $(n=25)$ & \\
\hline Gender $^{\mathrm{a}}$ & & & 0.059 \\
Female & $21(84.0 \%)$ & $15(60.0 \%)$ & \\
Male & $4(16.0 \%)$ & $10(40.0 \%)$ & \\
Age $(\text { years })^{\mathrm{b}}$ & $29.88 \pm 6.28$ & $29.88 \pm 6.43$ & 1.000 \\
Height $(\mathrm{cm})^{\mathrm{b}}$ & $163.42 \pm 5.91$ & $167.27 \pm 10.73$ & 0.125 \\
Weight $(\mathrm{kg})^{\mathrm{b}}$ & $112.42 \pm 17.39$ & $127.93 \pm 25.58$ & $0.016^{*}$ \\
BMI (kg/m $)^{\mathrm{b}}$ & $41.91 \pm 5.09$ & $45.31 \pm 5.33$ & $0.026^{*}$ \\
Comorbid conditions & & & \\
Hyperlipidemia & & 0.560 \\
Nonalcoholic steatohepatitis $^{\mathrm{a}}$ & $8(32.0 \%)$ & $8(32.0 \%)$ & 0.154 \\
Hypertension $^{\mathrm{a}}$ & $5(20.0 \%)$ & $14(56.0 \%)$ & 0.130 \\
Diabetes mellitus $^{\mathrm{a}}$ & $5(20.0 \%)$ & $11(44.0 \%)$ & 0.700 \\
Hyperuricemia $^{\mathrm{a}}$ & $4(16.0 \%)$ & $3(12.0 \%)$ & 0.724 \\
Gallstones $^{\mathrm{c}}$ & $1(4.0 \%)$ & $6(24.0 \%)$ & 0.470 \\
Osteoarthritis $^{\mathrm{c}}$ & $1(4.0 \%)$ & $1(4.0 \%)$ & 1.000 \\
\hline
\end{tabular}


Table 3 A comparison of surgical results in 50 patients
SITU single-incision transumbilical, $S D$ standard deviation, $E W L$ excess weight loss

$* P<0.05$ (statistically significant difference between the two groups)

${ }^{\text {a }}$ Independent $t$ test was used
Surgical method

$P^{\mathrm{a}}$

SITU-LRYGB $(n=25) \quad$ 5-port LRYGB $(n=25)$

Mean \pm SD

\begin{tabular}{lccc}
\hline Operation time (min) & $99.8 \pm 11.14$ & $67.56 \pm 20.52$ & $<0.001^{*}$ \\
Hospital stay (days) & $1.12 \pm 0.43$ & $1.08 \pm 0.27$ & 0.702 \\
Frequency of morphine injections & $2.56 \pm 2.06$ & $1.68 \pm 1.28$ & 0.078 \\
1st EWL (\%) & $19.55 \pm 6.26$ & $19.20 \pm 6.02$ & 0.843 \\
3rd EWL (\%) & $40.19 \pm 9.21$ & $38.96 \pm 9.27$ & 0.641 \\
6th EWL (\%) & $54.92 \pm 8.87$ & $51.57 \pm 14.59$ & 0.332 \\
9th EWL (\%) & $65.98 \pm 10.17$ & $68.36 \pm 12.07$ & 0.465 \\
12th EWL (\%) & $70.86 \pm 11.75$ & $78.20 \pm 13.59$ & 0.225 \\
Wound satisfaction score & $4.48 \pm 0.58$ & $3.96 \pm 0.67$ & $0.006^{*}$ \\
\hline
\end{tabular}

$56 \%$ in the five-port group), and hypertension (20\% in the SITU group and $44 \%$ in the five-port group). However, compared to patients in the LRYGB group, the body weights (112.42 vs. $127.93 \mathrm{~kg}$, respectively; $P=0.016)$ and BMIs (41.91 vs. $45.31 \mathrm{~kg} / \mathrm{m}^{2}$, respectively; $P=0.026$ ) were significantly lower among patients who were treated with the single-incision approach.

The differences in operative results between the two groups are listed in Table 3. The length of postoperative hospitalization is no different between the two groups. Excess body weight loss (percent) in the first and third month in both groups was $19.5 \%$ and $19.1 \%, 40.1 \%$, and $42.7 \%$, respectively. The frequency of morphine injections in the SITU group appears to be greater than that in the five-port group, but this was not statistically significant. Although patients in the SITU group had longer operation times than those in the fiveport group (99.8 vs. $67.56 \mathrm{~min}$, respectively; $P<0.001$ ), the overall satisfaction score of the SITU group was significantly higher than that of the five-port group (4.48 vs. 3.96; $P=$ 0.006). That is, the patients with SITU-LRYGB were more satisfied with the outcome of their incisions (Fig. 3).

There were no complications in the two groups of patients. After umbilicoplasty, patients who had the SITULRYGB procedure needed to care for their wounds longer (2 to 3 weeks) than did those who had the five-port procedure (1 week). This was due to the fact that the SITULRYGB wound produces more tension during closure, compared to the wound closure of the five-port procedure.

\section{Discussion}

Recently, single-incision laparoscopic surgery has been gradually adopted in bariatric surgery. Saber et al. reported the first series of patients treated with single-incision laparoscopic sleeve gastrectomy [15]. This surgical technology offers an alternative to NOTES, which is still an experimental procedure and a frequent topic of debate [17, 18]. The internal surgery used for SILS and the multiport approach are not different, but the surgeon may experience hand-crossing problems during manipulation of the instruments. Using multiple transfascial punctures in SITU procedures to access the abdominal cavity is similar to the conventional trocar insertion technique except that the ports are colocated in a smaller space. All layers except the skin and subcutaneous fat are pierced. And to decrease the possibility of future periumbilical herniation, the fascial punctures need to be sutured during the SITU procedure. Because the surgery is done transumbilically and the wound is embedded in the umbilicus, the surgical scar can be almost completely hidden inside the belly button, and there is minimal scarring. Although some might argue that very obese patients do not care about scarring, up to $70 \%$ of our patients are female, and they are concerned about scarring after the weight loss. The surgery is optional, and indeed, some patients choose the SITU approach.

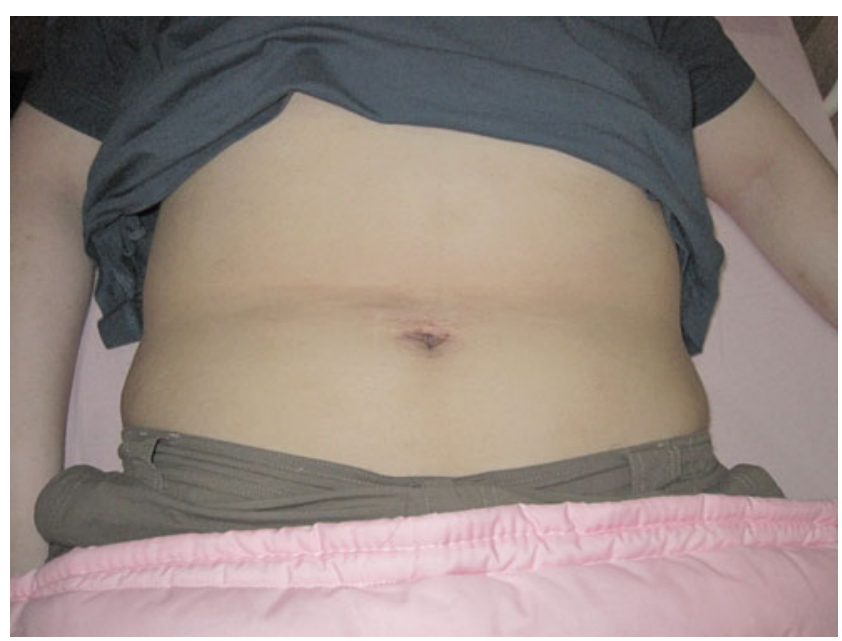

Fig. 3 Cosmetic outcome of a SITU wound after umbilicoplasty 
In the past, the main barrier to performing this surgery has been the crowding of trocars in a very limited surgical field. And previously, the resulting small degree of instrument triangulation and lack of tissue retraction by the assistant surgeon have made the procedure arduous. In addition, handling a hypertrophic liver is critical in morbidly obese patients.

When we first performed the first single-incision transumbilical LRYGB, using the 4-cm omega wound without subsequent umbilicoplasty, it took us $180 \mathrm{~min}$ to finish the procedure [19]. In subsequent patients, we enlarged the omega-shaped incision slightly to $6 \mathrm{~cm}$. Using a 6-cm omega-shaped incision allows more space between trocars and also permits the surgeon to more easily move these instruments. We used conventional trocars to help surgeons use their conventional instruments to finish the procedure, while some may be concerned that use of the SITU procedure, which is performed without articulating instruments, might compromise the angles of the gastric incision used to form the pouch. The distance was $4 \mathrm{~cm}$ more between trocars with our design, so no articulating instrument was needed. However, articulating linear cutting staplers were still necessary to create the gastric pouch and anastomosis. And the 6-cm incision wound around the umbilicus can be revised with umbilicoplasty to become a $3.5-\mathrm{cm}$ circular wound. Recently, we have used special trocars such as the Dexide ${ }^{\mathrm{TM}} 5-\mathrm{mm}$ threaded trocar (Covidien, USA) and shortened the incision to $4 \mathrm{~cm}$. In the future, using this new instrument, it may be possible to shorten the incision and thus to decrease the patient's pain and further improve cosmetic result. And because of the longer distance between the gastric pouch and umbilical port than five-port surgery, we used $43-\mathrm{cm}$ instruments, including endoscope, graspers, and XL-sized GIA $(10 \mathrm{~cm}$ longer than the conventional type) to overcome the problems in SITU procedures.

Patient selection is important when considering the SITU procedure. Some patients are not well suited for it; for example, we will not perform the procedure for super-obese patients, or those with BMIs greater than 60 . Patients taller than $180 \mathrm{~cm}$ are also poor candidates for the procedure because the abundant fat in the abdomen and the very long distance between the umbilicus and the gastric pouch make the surgery very difficult. And the procedure does involve a learning curve. We recommend that the procedure be performed only by experienced bariatric surgeons; for example, the primary surgeon had completed more than 400 laparoscopic Roux-en-Y gastric bypasses, so the technique could be smoothly performed.

The procedure also involves puncture of the liver, although we have observed no side effects from this. Blood loss from liver retraction was negligible and in fact so slight that it could not be quantified. There was no measurable blood loss with the liver suspension technique, and no subsequent hemorrhage or liver biloma or abscess formation was reported at follow-up. While surgical exposure is not as good as with a conventional mechanical liver retractor, the approach still provides enough space for the SITU procedure. However, some surgeons are still concerned about injury to the liver, and we need to find a better method for retracting the liver without harming it. Other considerations when choosing SITU-LRYGB include the challenges of establishing counter-traction and the need for surgical modifications, including the use of the liver suspension tape and the need for stay traction for jejunojejunostomal repair. The main interoperative advantage of the five-port LRYGB is that the assistant can help with counter-traction, and it is easier to perform the handsewn technique.

To our knowledge, this is the first case series in the literature describing single-incision laparoscopic Roux-en$\mathrm{Y}$ gastric bypass. The novel technique of using liver suspension tape for intraoperative liver retraction makes the operation process easier and eliminates the need for another hand to "push" the liver. With these modifications, the surgery can usually be completed in 120 min without the need for specially designed flexible articulating instruments or an endoscope, beyond the conventional straight laparoscopic instruments. As this technique is improved and more widely understood, it may well be applied to more advanced and complicated types of surgery that involve anastomosis of the gastrointestinal tract.

In conclusion, SITU-LRYGB is a safe, technically feasible, and reproducible procedure for gastric bypass for the majority of morbidly obese patients. And with the rapid development of more flexible articulating instruments, endoscopes, and robotic assistance, this approach undoubtedly will soon have wider applications. We have begun prospectively randomized studies to compare SITULRYGB with five-port LRYGB.

\section{Acknowledgments None.}

Conflict of interest The authors have no conflict of interest.

Sources of support None.

Open Access This article is distributed under the terms of the Creative Commons Attribution Noncommercial License which permits any noncommercial use, distribution, and reproduction in any medium, provided the original author(s) and source are credited.

\section{References}

1. Sugerman HJ, Kellum JM, Engle KM, et al. Gastric bypass for treating severe obesity. Am J Clin Nutr. 1992;55:560S-6. 
2. Benotti PN, Forse RA. The role of gastric surgery in the multidisciplinary management of severe obesity. Am J Surg. 1995;169:361-7.

3. Pories WJ, MacDonald Jr KG, Morgan EJ, et al. Surgical treatment of obesity and its effect on diabetes: 10-y follow up. Am J Clin Nutr. 1992;55:582S-5.

4. Demaria EJ, Jamal MK. Surgical options for obesity. Gastroenterol Clin N Am. 2005;34:127-42.

5. Schauer PR, Ikramuddin S, Hamad G, et al. Laparoscopic gastric bypass surgery: current technique. J Laparoendosc Adv Surg Tech Part A. 2003;13:229-39.

6. Wittgrove AC, Clark GW, Tremblay LJ. Laparoscopic gastric bypass, Roux-en-Y: preliminary report of five cases. Obes Surg. 1994;4:353-7.

7. Huang CK, Lee YC, Hung CM, et al. Laparoscopic Roux-en-Y gastric bypass for morbidly obese Chinese patients: learning curve, advocacy and complications. Obes Surg. 2008;18:77681

8. de la Fuente SG, Demaria EJ, Reynolds JD, et al. New developments in surgery: Natural Orifice Transluminal Endoscopic Surgery (NOTES). Arch Surg. 2007;142:295-7.

9. McGee MF, Rosen MJ, Marks J, et al. A primer on natural orifice transluminal endoscopic surgery: building a new paradigm. Surg Innov. 2006;13:86-93.

10. Flora ED, Wilson TG, Martin IJ, et al. A review of natural orifice translumenal endoscopic surgery (NOTES) for intra-abdominal surgery: experimental models, techniques, and applicability to the clinical setting. Ann Surg. 2008;247:583-602.
11. Martínez AP, Bermejo MA, Cortś JC, et al. Appendectomy with a single trocar through the umbilicus: results of our series and a cost approximation. Cir Pediátr. 2007;20:10-4.

12. Tacchino R, Greco F, Matera D. Single-incision laparoscopic cholecystectomy: surgery without a visible scar. Surg Endosc. 2009;23:896-9.

13. Cuesta MA, Berends F, Veenhof AA. The "invisible cholecystectomy": A transumbilical laparoscopic operation without a scar. Surg Endosc. 2008;22:1211-3.

14. Reavis KM, Hinojosa MW, Smith BR, et al. Single-laparoscopic incision transabdominal surgery sleeve gastrectomy. Obes Surg. 2008;18:1492-4.

15. Saber AA, Elgamal MH, Itawi EA, et al. Single incision laparoscopic sleeve gastrectomy (SILS): a novel technique. Obes Surg. 2008;18:1338-42.

16. Nguyen NT, Hinojosa MW, Smith BR, et al. Single laparoscopic incision transabdominal (SLIT) surgery-adjustable gastric banding: a novel minimally invasive surgical approach. Obes Surg. 2008;18:1628-31.

17. Bessler M, Milone L. Hybrid NOTES sleeve gastrectomy in an animal model. In: Proceedings of the P120 American Society for Metabolic and Bariatric Surgery Meeting, Washington, DC, 2008.

18. Mintz Y, Horgan S, Savu MK, et al. Hybrid natural orifice translumenal surgery (NOTES) sleeve gastrectomy: a feasibility study using an animal model. Surg Endosc. 2008;22:1798-802.

19. Huang CK, Houng JY, Chiang CJ, et al. Single incision transumbilical laparoscopic Roux-en-Y gastric bypass: a first case report. Obes Surg. 2009;19(12):1711-5. 